\title{
Histological similarity between tubulointerstitial nephritis and salivary gland biopsy in primary Sjögren's syndrome
}

\author{
Yoon Ji Tak ${ }^{1}$, Jong Sun Kim', Kyung-Ann Lee ${ }^{1}$, Hyun-Sook Kim', and So-Young Jin ${ }^{2}$
}

Departments of ${ }^{1}$ Internal Medicine and ${ }^{2}$ Pathology, Soonchunhyang University Seoul Hospital, Soonchunhyang University College of Medicine, Seoul, Korea
Received : July 15, 2021

Revised : July 30, 2021

Accepted: August 5, 2021

\section{Correspondence to} Hyun-Sook Kim, M.D.

Tel: +82-2-710-3214

Fax: +82-2-709-9554

E-mail: healthyra@schmc.ac.kr https://orcid.org/0000-0001-92137140
Primary Sjögren's syndrome (pSS) is characterized by dry eyes and mouth due to the lymphocytic inflammation of exocrine glands. Despite the wide spectrum of clinical manifestations, renal involvement is uncommon in pSS, and renal dysfunction is rarely present at diagnosis. The most common renal manifestation is tubulointerstitial nephritis (TIN), characterized by mononuclear lymphocytic inflammation (with more CD4+ than CD8+ T cells) in the interstitium.

A 60-year-old woman presented with acute renal dysfunction. She did not have a history of dry eyes, arthritis, or Raynaud's phenomenon. A speckled cytoplasmic pattern with 1:1,280 titer was noted with antinuclear antibodies. The level of rheumatoid factor was 2,389 $\mathrm{IU} / \mathrm{mL}$ (range, 0

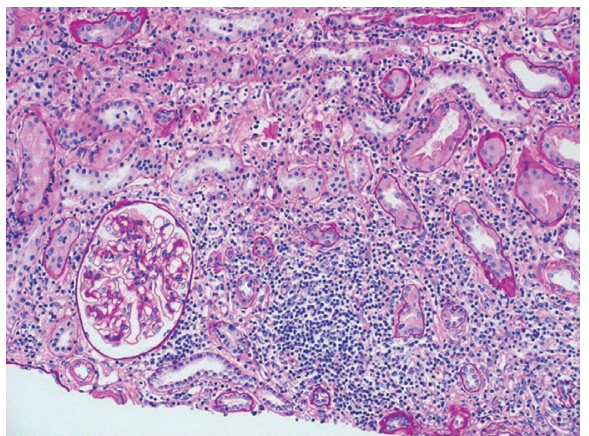

Figure 1. Renal biopsy showed marked interstitial inflammation by lymphoplasma cells and normal-appearing glomerulus (Periodic acid-Schiff stain, $\times 200$ ). to 14), and anti-cyclic citrullinated peptide antibody was undetectable. Anti-Ro antibodies (3+), anti-La antibodies (1+), and serum immunoglobulin $\mathrm{G}(1,848 \mathrm{mg} / \mathrm{dL}$; range, 700 to 1,600$)$ were positive. Anti-ds-DNA, anti-Sm, anti-cardiolipin, and anti-phospholipid antibodies, antineutrophil cytoplasmic antibodies, and cryoglobulin were negative. Renal biopsy showed acute immune-mediated TIN, without significant glomerular immunofluorescence staining (Fig. 1). Despite no complaints of dry mouth, the unstimulated salivary flow rate in this patient was $0 \mathrm{~mL} / \mathrm{min}$. Salivary gland ultrasonography exhibited multiple inhomogeneous hypoechoic areas with increased vascularity. Minor labial salivary gland biopsy showed focal lymphocytic sialadenitis (focus score, $4 / 4 \mathrm{~mm}^{2}$ ) (Fig.

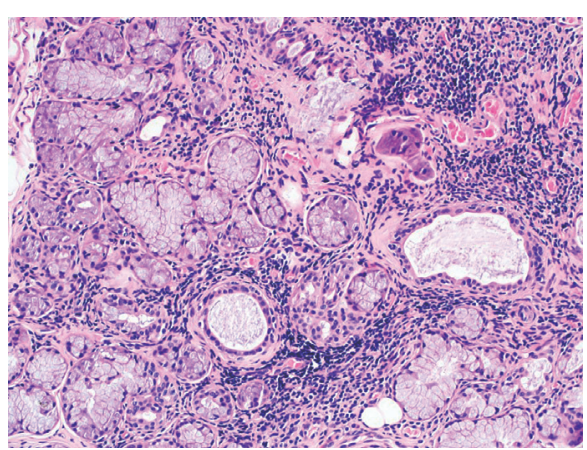

Figure 2. Minor salivary gland biopsy revealed multifocal interstitial inflammation by lymphoplasma cells (H\&E, ×200; focus score, $4 / 4 \mathrm{~mm}^{2}$ ). 
2). The patient was administered prednisolone (1 mg/ $/ \mathrm{kg}$ ), which was tapered over 2 weeks.

Monolymphocytic TIN can cause interstitial fibrosis. Similar to findings from salivary gland biopsy, plasma cell inflammation was observed in the renal interstitium. Dry eyes and articular symptoms were observed less frequently in pSS patients with renal involvement. Therefore, pSS is difficult to diagnose in patients who present with renal dysfunction as the first manifestation of pSS. Both renal and salivary gland biopsy should be considered for pSS patients with suspected renal involvement. This study was approved by the Insti- tutional Review Board of Soonchunhyang University Seoul Hospital (2021-07-016).

\section{Conflict of interest}

No potential conflict of interest relevant to this article was reported.

\section{Acknowledgments}

This study is supported by fund of the Soonchunhyang University. 